\title{
ESTUDIO DEL IMPACTO EN LA CALIDAD DEL AIRE DE LAS FUENTES PUNTUALES EN LA CIUDAD DE PINAR DEL RÍO.
}

\author{
MARIAM FONSECA HERNANDEZ
}

\author{
Centro de Contaminación y Química Atmosférica, Instituto de Meteorología de Cuba (INSMET), La \\ Habana, Cuba \\ mariam.fonseca@insmet.cu
}

Recibido Febrero de 2012 - Aceptado Julio de 2012

\begin{abstract}
RESUMEN
Se realizó un estudio del impacto de las fuentes fijas en la Ciudad de Pinar del Río utilizando el sistema de modelación MM5-CALMET-CALPUFF. Se simuló la dispersión de $\mathrm{SO}_{2}, \mathrm{NO}_{\mathrm{x}}, \mathrm{PM}_{25}, \mathrm{PM}_{10}$ y $\mathrm{CO}$ emitidos por las 21 fuentes durante 15 días representativos del período lluvioso, además de la depositación húmeda de $\mathrm{SO}_{4}{ }^{2-}$ y $\mathrm{NO}_{3}{ }^{-}$. Se observó que las máximas concentraciones se producen en horarios de la mañana y noche. Se observó que producto de estas emisiones se ven afectados algunas zonas cercanas a la ciudad como Minas de Matahambre, San Juan y Martínez, Santa María, San Luis, El Corojo, Hermanos Saíz, Viñales, Pilotos, Consolación del Sur y las Ovas. Los valores de concentración medios diarios de $\mathrm{NOx}$ y $\mathrm{SO}_{2}$ sobrepasaron sus concentraciones máximas admisibles según la NC 39: 1999 y estuvieron por encima de los valores guías de la Organización Mundial de la Salud y los valores estándares EPA.

Palabras clave: concentraciones, deposición, CALPUFF, fuentes emisoras.
\end{abstract}

\begin{abstract}
A STUDY OF THE IMPACT OF STATIONARY SOURCES ON THE AIR QUALITY OF PINAR DEL RIO CITY

A study of the impact of stationary sources on air quality on the city of Pinar del Rio, Cuba, using the modeling system MM5-CALMET-CALPUFF was made. The dispersion of $\mathrm{SO}_{2}, \mathrm{NO}_{\mathrm{x}}, \mathrm{CO}, \mathrm{PM}_{10}$ and $\mathrm{PM}_{25}$ emitted from 21 sources during 15 days is simulated by the model. This period is representative of the rainy season in Cuba (Summer). The of $\mathrm{SO}_{4}{ }^{2-}$ and $\mathrm{NO}_{3}{ }^{-}$wet deposition was also simulated. It was observed that the highest concentrations occur in the morning and evening. These emissions affected some neighboring cities as Santa Maria and Viñales. The daily average concentration values of $\mathrm{NO}_{\mathrm{x}}$ and $\mathrm{SO}_{2}$ exceeded their maximum permissible concentrations according to the Cuban standards, the guideline values of the World Health Organization and the EPA standards. The southwest area of the city is the most affected one with the highest concentration level.
\end{abstract}

Keywords: concentrations, deposition, CALPUFF, emissions sources.

\section{INTRODUCCIÓN}

Los contaminantes atmosféricos son especies químicas que en concentraciones superiores a cierto valor pueden ser perjudiciales no solo para la salud humana, sino también para la salud animal y vegetal. El incremento de estas concentraciones está dado tanto por procesos naturales como por actividades antrópicas. Las emisiones de origen antropogénico juegan un papel cada vez más importante en los problemas de contaminación atmosférica ya que han experimentado un incremento gradual y sostenido desde la última mitad del siglo XX (WHO, 2000).
En los últimos tiempos se ha observado un detrimento de la calidad del aire principalmente en aquellas regiones densamente pobladas, provocado básicamente por un aumento substancial de las emisiones de contaminantes a la atmósfera (WHO, 2000). No obstante, mecanismos como el transporte, la transformación química, la dispersión y el depósito de contaminantes emitidos desde zonas industrializadas ocasionan el deterioro de la calidad del aire en zonas alejadas de las fuentes de emisión.

El municipio de Pinar del Río es el municipio cabecera de la provincia del mismo nombre en la isla de Cuba (Figura 1) y está situado en su porción sur, su centro se encuentra 
aproximadamente en $\operatorname{los} 22.4166^{\circ}$ de latitud norte y $83.7000^{\circ}$ de longitud oeste, abarca una extensión de 707,8 km², correspondiendo $691,13 \mathrm{~km}^{2}$ a tierra firme y $16,67 \mathrm{~km}^{2}$ a cayos, y tiene una densidad poblacional de 270,2 hab $/ \mathrm{km}^{2}$ (ONE, 2009). Esta ciudad tiene su base económica primariamente en los servicios y por ser una ciudad principal cuenta con un gran número de instalaciones, fundamentalmente industrias, por lo que resultaría de extrema importancia determinar el impacto sobre la calidad del aire de las fuentes puntuales ubicadas en ella, lo que constituye el objetivo principal de este estudio.

La modelación de la contaminación atmosférica es un intento de describir la relación funcional entre las emisiones y las concentraciones y deposiciones producidas (Builtjes, 2001). Los Modelos de Calidad del Aire utilizan técnicas matemáticas y numéricas para simular aquellos procesos físicos y químicos que sufren los contaminantes en la atmósfera. Para esto utilizan una serie de datos de entrada como datos meteorológicos, del terreno, de uso del suelo y de información técnica de la fuente emisora (coordenadas geográficas, tasas de emisión, altura y diámetro de la chimenea, etc.).

La simulación de los contaminantes asociados a las emisiones de estas fuentes fue realizada mediante la aplicación del sistema de modelación atmosférica "MM5-CALMETCALPUFF" definido por la agencia Agencia de Protección Ambiental de Estados Unidos (EPA) como sistema de referencia para simular la dispersión de emisiones provenientes de complejos industriales ubicados en terreno complejo.

Internacionalmente se han realizado una serie de estudios que utilizan el sistema de modelación CALMET-CALPUFF para evaluar el impacto de fuentes puntuales en la calidad del aire, como los realizados por Valdés-Manzanilla et al. (2008) en México donde fue determinado que se sobrepasa el valor recomendado por la Organización Mundial de la Salud para el $\mathrm{SO}_{2}$. También los realizados por Hao et al. (2006) en Beijing cuyos resultados mostraron que en el 2000 la contribución de las emisiones inducen una contribución relativamente pequeña a las concentraciones medias ambientales, con un impacto significativo en el área urbana en Beijing, China y además los estudios de Levy et al. (2001) en Illinois, Estados Unidos donde se encontró que la población afectada por el transporte de los contaminantes y el número de plantas de energía que fungen como fuentes en los EE.UU implica impactos en la salud que son potencialmente significantes.

En Cuba se han realizado infinidad de estudios sobre calidad del aire utilizando la modelación de contaminantes atmosféricos, entre estos se pueden citar Sánchez et al. (2004) sobre calidad del aire en el municipio Habana Vieja, La Habana y Collazo et al. (2004) sobre transformaciones químicas a escala local de $\mathrm{SO}_{4}{ }^{2-} \mathrm{y} \mathrm{NO}_{3}{ }^{-}$. También se han realizado varios estudios utilizando el sistema CALMET-CALPUFF como los realizados

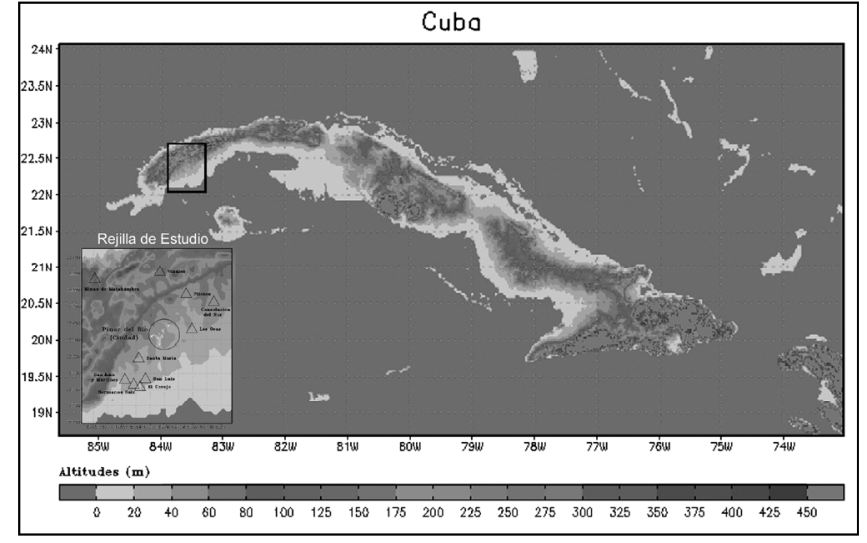

Figura 1 - Ubicación geográfica de la rejilla de estudio.

por González (2010) para la eficiencia y efectividad de los generadores terrestres de yoduro de plata ubicados en la isla, Fonseca (2010) para lograr su implementación y aplicación a escala local y Collazo (2011) para el análisis de la contaminación transfronteriza y local de los compuestos gaseosos precursores de la deposición ácida húmeda y formación de $\mathrm{O}_{3}$.

\section{MATERIALES Y MÉTODOS}

\subsection{Dominio de modelación}

Se obtuvo una rejilla de 60 kilómetros en la dirección zonal por 60 kilómetros en la dirección meridional con nodos que representan cuadrados de $1 \mathrm{~km}^{2}$ y con 10 niveles de altura $(0,20,40,80,160,320,640,1200,2000,3000$ y 4000 m). La rejilla de cálculo abarca toda la Ciudad de Pinar del Río y dentro de ella están ubicadas todas las fuentes simuladas como se muestra en la Figura 2, en ella se representa aproximadamente a la Ciudad de Pinar del Río con un círculo, los pueblos cercanos con triángulos y las fuentes con cruces.

El dominio de estudio está comprendido entre $22.1202^{\circ}$ - $22.7130^{\circ}$ de la latitud Norte $(\mathrm{N})$ y $83.4036^{\circ}-83.9964^{\circ} \mathrm{de}$ longitud Oeste (W). Los cálculos se realizaron a 21 fuentes puntuales en la Ciudad de Pinar del Río. Se utilizó la proyección geográfica Lambert Conformal Conic (LCC) con el dominio centrado en $\operatorname{los} 22.4166^{\circ}$ de latitud Norte y $83.7000^{\circ}$ de longitud Oeste, que representa un punto en el centro de la Ciudad de Pinar del Río.

\subsection{Datos geofísicos, meteorológicos, técnicos de las fuentes y días simulados}

El módulo geofísico incluye la información de la topografía y el uso del suelo. Ambos datos fueron descargados de la página web del Servicio Geológico de Estados Unidos (United 


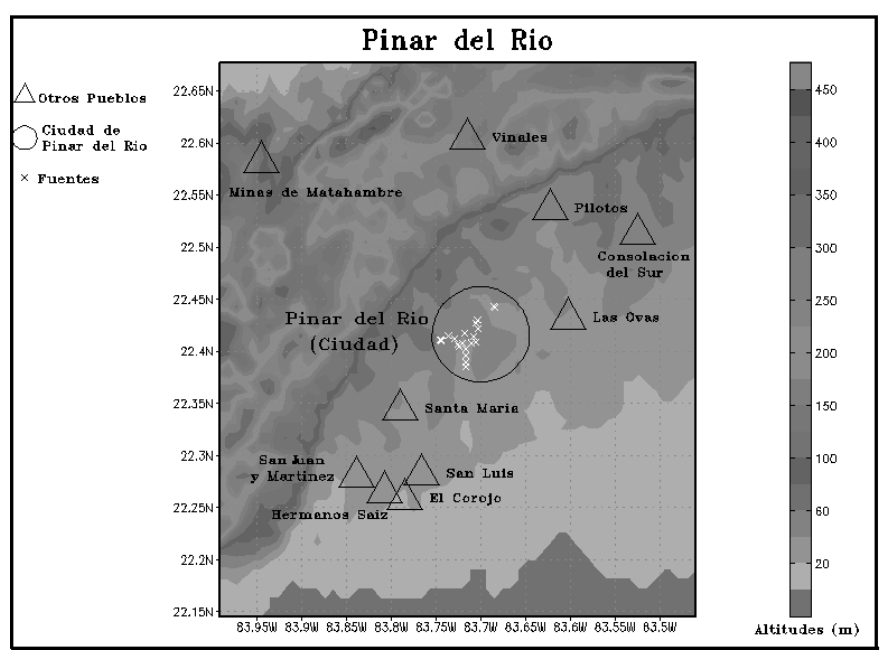

Figura 2 - Rejilla de estudio y fuentes puntuales.

States Geological Survey USGS). Los datos meteorológicos se obtuvieron del modelo MM5V3 (Mesoscale Model 5 Version 3) con una resolución espacial de $9 \mathrm{Km}$ y cada 1 hora. Se utilizaron salidas del MM5V3 las cuales comienzan a las $00 \mathrm{Z}$ y comprenden un período de 72 horas, de estas salidas se utilizaron para las corridas solo 24 horas (horas de las 6 a la 30). Fueron simulados 15 días, todos del período lluvioso. La simulación de realizó utilizando un período de tiempo de 24 horas, aunque en algunas ocasiones se cuentan con días consecutivos las corridas se hicieron independientes para cada uno de estos días. Todos los datos técnicos de las fuentes utilizados fueron los obtenidos por Rodríguez et al., (2007).

\subsection{Sistema de modelación CALMET-CALPUFF}

El sistema de modelación CALMET-CALPUFF está constituido por un modelo meteorológico en el cual están agrupados un módulo de campo de vientos diagnóstico y módulos micrometeorológicos para las capas límite sobre agua y sobre tierra y un modelo que simula los efectos del tiempo, del espacio y la variación de las condiciones meteorológicas en el transporte de contaminantes, su transformación y remoción.

\subsubsection{Algunas opciones utilizadas en la simulación}

- Las salidas del MM5 fueron utilizadas como datos de estaciones en superficie, en agua y de aire superior.

- Se usó el campo de vientos de las salidas del MM5 como campo de vientos inicial.

- Cantidad de especies químicas simuladas: $8\left(\mathrm{NO}_{\mathrm{X}}\right.$, $\mathrm{HNO}_{3}, \mathrm{NO}_{3}^{-}, \mathrm{SO}_{2}, \mathrm{SO}_{4}{ }^{2-}, \mathrm{CO}, \mathrm{PM}_{10}$ y $\mathrm{PM}_{25}$ )

- Cantidad de especies emitidas: $5\left(\mathrm{NO}_{\mathrm{x}}, \mathrm{SO}_{2}, \mathrm{CO}\right.$, $\mathrm{PM}_{10}$ y $\left.\mathrm{PM}_{25}\right)$.
- Cantidad de fuentes puntuales: 21. (Todas las fuentes tienen emisiones variables, la mayoría de estas comienzan a emitir entre las 6 am -8 am, solo una de ellas emite 24 horas)

- Método utilizado para el ajuste del terreno: Ajuste CALPUFF.

- Cálculo de los valores de los coeficientes de dispersión mediante los valores internos calculados de turbulencia usando variables micrometeorológicas obtenidas de CALMET.

- Esquema MESOPUFF II como mecanismo de transformaciones químicas.

Algunas variables que influyen en el mecanismo de transformaciones químicas:

- Tasas de conversión nocturnas (se utilizaron los valores predefinidos)

- Concentraciones de fondo de $\mathrm{O}_{3}$ y $\mathrm{NH}_{3}$ (Tabla 1). Datos obtenidos de los trabajos realizados por Fonseca (2010) y Ramírez (1989).

\subsubsection{Concentraciones de fondo}

Por el esquema químico seleccionado, CALPUFF necesita las concentraciones de fondo de $\mathrm{NH}_{3}$ (Fonseca $\mathrm{M}$, 2010) y $\mathrm{O}_{3}$ (Ramírez J, 1989) correspondientes al dominio de modelación, este emplea valores espacialmente uniformes, pero es posible separarlos por meses del año.

La oxidación de $\mathrm{NO}_{2}$ a $\mathrm{HNO}_{3}$ por $\mathrm{OH}$ durante el día y de $\mathrm{NO}_{3}{ }^{-}$por $\mathrm{O}_{3}$ en la noche es el proceso de eliminación más importante de los $\mathrm{NO}_{\mathrm{x}}$ en la atmósfera. El MESOPUFF II usa las concentraciones de fondo de $\mathrm{O}_{3}$ (junto con la intensidad de la radiación) como sustitutos para la concentración de $\mathrm{OH}$ durante el día. Para el $\mathrm{O}_{3}$ y $\mathrm{NH}_{3}$ se utilizaron las concentraciones mensuales que aparecen en la Tabla 1:

\subsection{Concentraciones máximas admisibles}

La Organización Mundial de la Salud (OMS) tiene guías de calidad del aire que tienen por objeto ofrecer orientación

Tabla 1 - Concentraciones mensuales de fondo de $\mathrm{O}_{3}$ y NH $\mathrm{NH}_{3}(\mathrm{ppb})$.

\begin{tabular}{|l|l|l|l|l|l|}
\hline Meses & $\mathbf{O}_{3} \cdot$ & $\mathbf{N H}_{3} \cdot$ & Meses & $\mathbf{O}_{3} \cdot$ & $\mathbf{N H}_{3} \cdot$ \\
\hline$\underline{\text { Enero }}$ & 74.77 & 1.480 & $\underline{\text { Julio }}$ & 16.36 & 5.410 \\
\hline$\underline{\text { Febrero }}$ & 67.76 & 2.770 & $\underline{\text { Agosto }}$ & 15.42 & 1.450 \\
\hline$\underline{\text { Marzo }}$ & 74.77 & 1.540 & $\underline{\text { Septiembre }}$ & 19.63 & 3.910 \\
\hline$\underline{\text { Abril }}$ & 18.69 & 1.640 & $\underline{\text { Octubre }}$ & 38.79 & 3.550 \\
\hline$\underline{\text { Mayo }}$ & 8.88 & 1.230 & $\underline{\text { Noviembre }}$ & 55.14 & 2.850 \\
\hline$\underline{\text { Junio }}$ & 11.68 & 1.320 & $\underline{\text { Diciembre }}$ & 49.07 & 1.690 \\
\hline
\end{tabular}


sobre la manera de reducir los efectos de la contaminación del aire en la salud. Los niveles establecidos por la OMS no son una norma, son unas directrices para ayudar a los países a obtener su propio estándar de calidad del aire, con el objeto de ayudar a proteger la salud humana de la contaminación del aire. La Tabla 2 muestra un compendio de las CMA según la NC 39:1999, las recomendaciones de la OMS y los valores regulatorios de la EPA, valores con los cuales serán comparados los resultados obtenidos.

\subsection{Metodología de análisis}

Para poder determinar el impacto de las fuentes fijas en la Ciudad de Pinar del Río se analizaron los máximos horarios y los máximos de 24 horas para poder determinar si las concentraciones encontradas exceden los valores guías para cada una de las especies simuladas, entiéndase por máximos horarios el mayor valor de todos los máximos registrados para períodos de 1 hora, y los máximos de 24 horas son los mayores valores registrados pero para períodos de 24 horas. Se determinan las posiciones de los máximos que sobrepasen los valores normados con el objetivo de conocer los lugares más afectados por las emisiones de estas fuentes y por lo tanto los más proclives a episodios de mala calidad del aire. Se utilizó el lenguaje de programación Fortran 90 para crear un programa que leyera los archivos de salida horarias y hallara los valores medios en la rejilla para cada una de estas especies y para cada uno de los días simulados para poder determinar el comportamiento medio diario de las especies simuladas con el objetivo de identificar patrones horarios de concentraciones medias en toda la malla. Además se procesaron de igual forma las salidas de algunas variables meteorológicas como la temperatura, las precipitaciones y la fuerza y dirección del viento para encontrar relaciones entre el comportamiento de estas y los resultados obtenidos. Se expresan en por cientos la cantidad de días en que se sobrepasan los valores guías como un método para mostrar los resultados, no como una herramienta estadística.

\subsubsection{Métodos y herramientas utilizadas para el procesamiento de los resultados}

Las gráficas fueron realizadas con GRADS y todos los programas realizados para el post-procesamiento de las salidas se hicieron en FORTRAN.

\section{ANÁLISIS DE LOS RESULTADOS}

\subsection{Comportamiento medio horario}

Para el conjunto de días estudiados se obtuvieron los valores medios horarios para cada especie química simulada, en los cuales se pueden apreciar dos patrones bien definidos. El primero de ellos determinado por el $\mathrm{SO}_{2}$ y $\mathrm{NO}_{\mathrm{x}}$, con rango de variabilidad horaria entre 0 y $0,6 \mu \mathrm{g} / \mathrm{m}^{3}$ y 0 y $0,3 \mu \mathrm{g} / \mathrm{m}^{3}$ respectivamente. En la Figura 3 se muestra el comportamiento medio horario de ambas especies, en el cual se observa un máximo absoluto alrededor de las 8 am cercano a los $0,25 \mu \mathrm{g} /$ $\mathrm{m}^{3}$ en el caso de los $\mathrm{NO}_{\mathrm{x}}$ y cercano a los $0,5 \mu \mathrm{g} / \mathrm{m}^{3}$ para el

Tabla 2 - Concentraciones máximas admisibles según la Norma Cubana 39:1999, las recomendaciones de la OMS según la actualización mundial del 2005 y los estándares EPA, en $\mu \mathrm{g} / \mathrm{m}^{3}$.

\begin{tabular}{|c|c|c|c|c|}
\hline Contaminante. & Período de tiempo & NC:39 (1999) & OMS (2005) & EPA \\
\hline \multirow{2}{*}{$\mathrm{SO}_{2}$} & 1 Hora & 250 & & 196 \\
\hline & 24 Horas & 50 & 20 & \\
\hline \multirow{2}{*}{$\mathrm{NO}_{2}$} & 1 Hora & 160 & 200 & 188 \\
\hline & 24 Horas & 40 & & \\
\hline \multirow{2}{*}{$\mathrm{CO}$} & 1 Hora & 24000 & & 40000 \\
\hline & 24 Horas & 3000 & & \\
\hline \multirow{2}{*}{$P M_{10}$} & 1 Hora & 200 & & \\
\hline & 24 Horas & 60 & 50 & 150 \\
\hline \multirow{2}{*}{$\mathbf{P M}_{25}$} & 1 Hora & 120 & & \\
\hline & 24 Horas & & 25 & 35 \\
\hline
\end{tabular}

Los valores para períodos de 1 hora para Cuba no constituyen una norma sino una propuesta para la nueva norma cubana de nuevas concentraciones máximas admisibles (Molina, 2010) que aún no han sido aprobadas. 


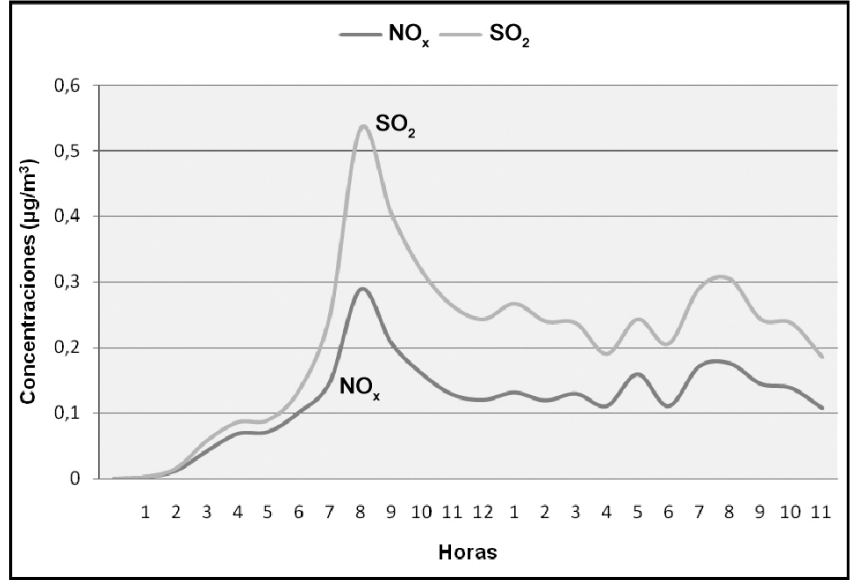

Figura 3 - Comportamiento medio horario de las concentraciones de $\mathrm{NO}_{\mathrm{x}}$ y $\mathrm{SO}_{2}$ desde las 12 am (hora 0) hasta las $11 \mathrm{pm}$ en todo el dominio de estudio.

$\mathrm{SO}_{2}$. En las horas posteriores presentan un comportamiento oscilatorio alrededor de los 0,1 y $0,2 \mu \mathrm{g} / \mathrm{m}^{3} \operatorname{los} \mathrm{NO}_{\mathrm{x}}$ y entre 0,2 $\mu \mathrm{g} / \mathrm{m}^{3}$ y $0,3 \mu \mathrm{g} / \mathrm{m}^{3}$ el SO $\mathrm{S}_{2}$ encontrándose otro máximo relevante aunque menor entre las $7 \mathrm{pm}$ y las $8 \mathrm{pm}$ para ambas especies, con valores de $0,18 \mu \mathrm{g} / \mathrm{m}^{3}$ en el caso de $\operatorname{los} \mathrm{NO}_{\mathrm{x}}$ y $0,31 \mu \mathrm{g} / \mathrm{m}^{3}$ el $\mathrm{SO}_{2}$. Ambas especies presentan una tendencia a disminuir en las últimas horas.

El segundo patrón corresponde al resto de las especies simuladas, que presentan una tendencia a aumentar las concentraciones medias en las primeras horas del plazo analizado con un máximo alrededor de las $7 \mathrm{am}$. En las horas posteriores la tendencia es a disminuir, presentándose un mínimo alrededor de las $4 \mathrm{pm}$, para luego volver a incrementar hacia las últimas horas del plazo estudiado, contrariamente a lo que se observa para el $\mathrm{SO}_{2}$ y $\mathrm{NO}_{\mathrm{x}}$. (Ver Figura 4). En cuanto al comportamiento de los valores medios horarios de deposición seca en la Figura 5 se aprecia que las mayores deposiciones ocurren entre las 7 am y 8 am, en correspondencia con los máximos de concentraciones medias de estas especies, algo bastante lógico, ya que a mayores valores de concentraciones es de esperar mayores valores de deposiciones. Sin embargo, resulta interesante que en el caso del $\mathrm{PM}_{10}$ se aprecie una tendencia a la disminución de las deposiciones en las últimas horas cuando sus concentraciones tienen una tendencia al incremento, lo cual puede estar determinado por la relación entre los flujos de deposición con otros factores como la estabilidad atmosférica, la cantidad de movimiento, el diámetro de las partículas, su aglomeración y densidad entre otros. (Sehmel, 1980).

Los valores medios horarios de deposición húmeda mostrados en las Figuras 6 y 7 muestran máximos en el horario de la tarde entre la $1 \mathrm{pm}$ y $6 \mathrm{pm}$, que corresponden con los horarios de mayor monto de precipitación.
Es de notar que los máximos de deposición húmeda ocurren en los mismos horarios en los que se reportan los mínimos de las concentraciones medias horarias (alrededor de las 4 pm) como se observa en la Figura 4 evidenciando así la contribución de la remoción húmeda al proceso de disminución de las concentraciones de estas especies.

\subsection{Comportamiento medio diario}

Durante los 15 días simulados el comportamiento medio diario de las concentraciones de los contaminantes simulados mantuvo un patrón similar. Generalmente se ve afectada toda la rejilla aunque los mayores valores se reportan en el centro, es decir, en la misma ciudad. Cuando esto no sucede, solo la parte oeste de la rejilla queda afectada con las concentraciones. Un ejemplo de esto se ve en la Figura 8.

De estas gráficas se puede inferir que no solamente la ciudad de Pinar del Río se ve afectada por las contribuciones de estas fuentes puntuales sino también algunos poblados aledaños a la ciudad. Por lo menos en los 15 días simulados en todas las gráficas de comportamiento medio diario se ven afectados además de la propia ciudad de Pinar del Río otras zonas como Minas de Matahambre, San Juan y Martínez, Santa María, San Luis, El Corojo y Hermanos Saíz; y en menor medida las zonas del norte y este de la ciudad, como Viñales, Pilotos, Consolación del Sur y las Ovas.

Las deposiciones secas y húmedas también presentan este patrón ya que durante los 15 días simulados prácticamente todos los días se ve afectada toda la rejilla y cuando esto no sucede solo se ve afectada la parte oeste y sur de la rejilla como se puede observar en las Figuras 9 y 10 siendo mayores los valores medios diarios de las deposiciones húmedas.

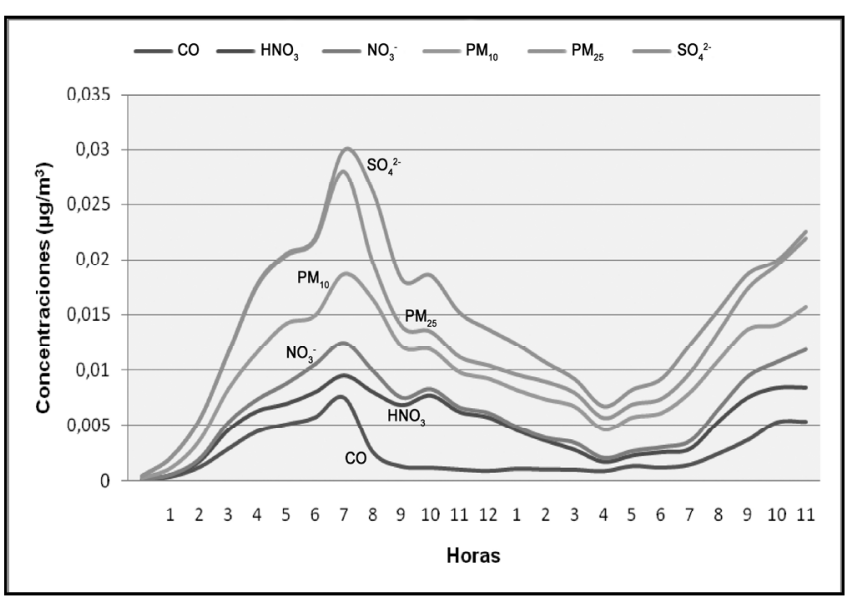

Figura 4 - Comportamiento medio horario de las concentraciones de $\mathrm{CO}, \mathrm{NO}_{3}{ }^{-}, \mathrm{PM}_{10}, \mathrm{PM}_{25} \mathrm{y} \mathrm{SO}_{4}{ }^{2-}$ desde las 12 am (hora 0 ) hasta las 11 $\mathrm{pm}$ en todo el dominio de estudio. 


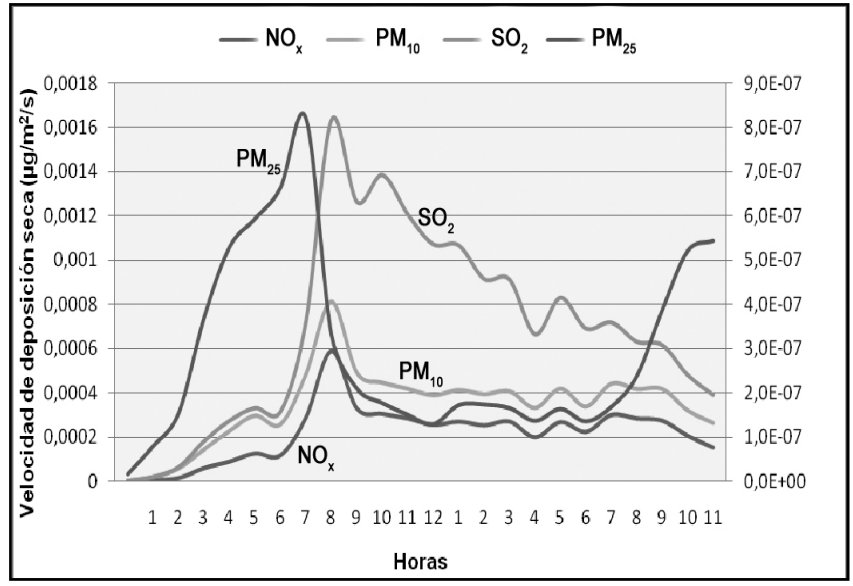

Figura 5 -Comportamiento medio horario de las deposiciones secas de $\mathrm{SO}_{2}, \mathrm{NO}_{\mathrm{x}}, \mathrm{PM}_{10}$ y $\mathrm{PM}_{25}$ desde las 12 am (hora 0) hasta las $11 \mathrm{pm}$. El eje secundario responde a los valores del $\mathrm{PM}_{25}$ en todo el dominio de estudio.

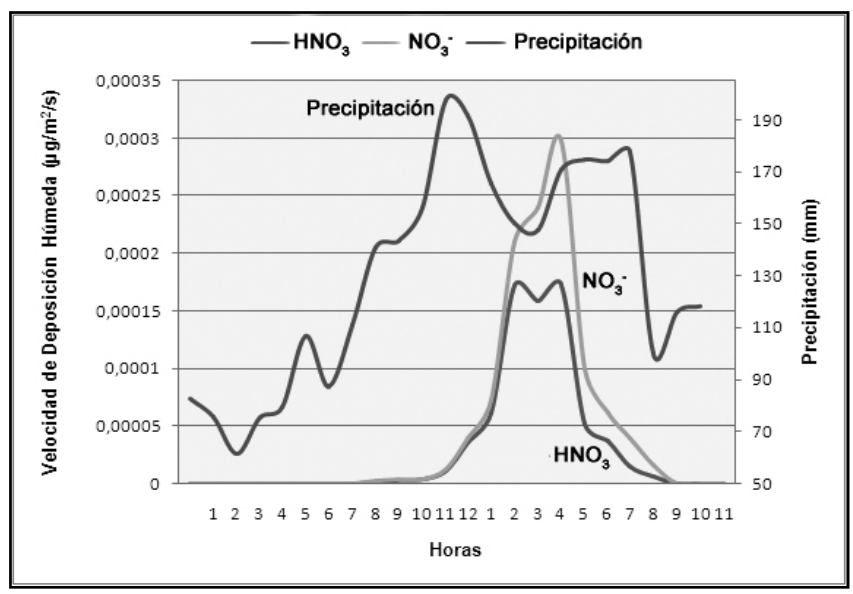

Figura 6 -Comportamiento medio horario de las deposiciones húmedas de $\mathrm{HNO}_{3}$ y NO$_{3}{ }^{-}$y de la precipitación media horaria (en mm) desde las 12 am (hora 0 ) hasta las $11 \mathrm{pm}$ en todo el dominio de estudio.

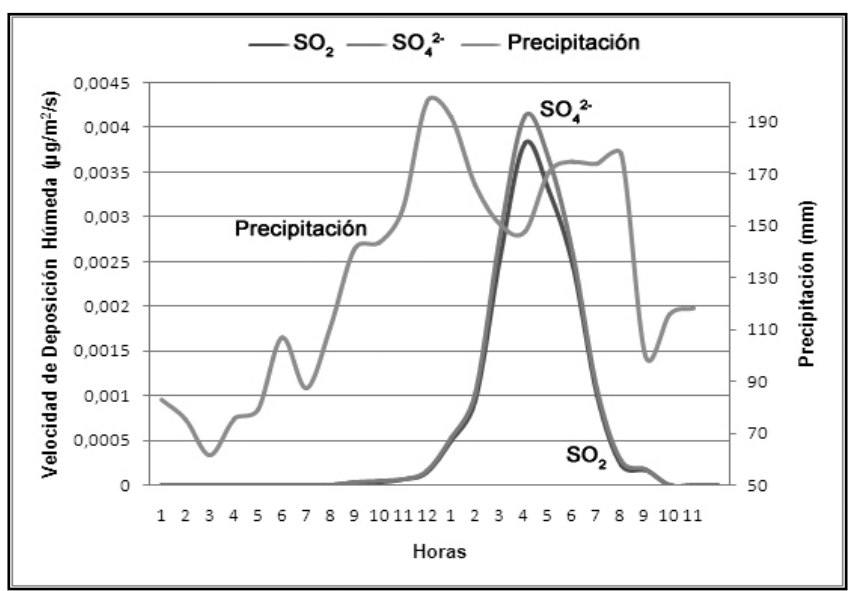

Figura 7 -Comportamiento medio horario de las deposiciones húmedas de $\mathrm{SO}_{2}$ y SO$_{4}{ }^{2-}$ y de la precipitación media horaria (en $\mathrm{mm}$ ) desde las $12 \mathrm{am}$ (hora 0 ) hasta las $11 \mathrm{pm}$ en todo el dominio de estudio.
Es notable que tanto los mayores valores de concentraciones y de deposiciones secas se presentan en el centro de la rejilla (donde están ubicadas las fuentes), sin embargo los mayores valores de deposiciones húmedas ocurren generalmente al oeste de esta, esto puede estar determinado por el carácter discreto de las precipitaciones que para los días estudiados presentaron una mayor tendencia a ocurrir en esta región.

Para los días estudiados el viento tuvo componente predominante del este, es por esto que generalmente se ve afectada toda la rejilla o solo la parte oeste de esta. En el primer caso, en el que se afecta toda la rejilla se puede observar a lo largo del día cómo el viento va girando del NE-S-N por lo que existen valores en prácticamente todos los puntos de la malla. En la Figura 11 se puede observar esta evolución.

Las gráficas en la Figura 11 representan el giro del viento del día 1ro de agosto del 2007. A la hora 0 el viento es predominante de NE, ya a las 8 am es dominante la componente del S comenzando a ser del SE a la $1 \mathrm{pm}$, de 5 pm a 6 pm comienza a girar del W al NW y finalmente a las $11 \mathrm{pm}$ es predominante del $\mathrm{N}$.

En el segundo caso en el que se ve afectada solo la parte oeste ocurre que el viento se mantiene con componente este a lo largo del día oscilando entre NE y SE. En la Figura 12 se puede observar como en las tres primeras horas que se muestran (hora 0,8 am y $1 \mathrm{pm}$ ) la dirección del viento presenta el mismo comportamiento que el día 1ro de agosto, sin embargo en las otras tres horas se mantiene con dirección predominante del NE por lo que la parte este de la rejilla no se ve afectada ya que el viento no llega a girar sino que se mantiene con componente E todo el tiempo.

\subsection{Valores medios diarios}

Los valores medios diarios de $\mathrm{NO}_{\mathrm{x}}$ oscilaron entre 0,001 $\mu \mathrm{g} / \mathrm{m}^{3}$ y $40 \mu \mathrm{g} / \mathrm{m}^{3}$ con máximos de hasta $92 \mu \mathrm{g} / \mathrm{m}^{3}$ y valores de deposición seca medios diarios entre $1 \mathrm{X}^{-8} 0^{-8} \mu \mathrm{g} / \mathrm{m}^{2} / \mathrm{s}$ y $1,0 \mu \mathrm{g} /$ $\mathrm{m}^{2} / \mathrm{s}$. Los valores de concentración diarias de $\mathrm{HNO}_{3}$ en los 15 días simulados oscilaron entre 0,001 y $2 \mu \mathrm{g} / \mathrm{m}^{3}$ con deposiciones húmedas medias diarias de entre 0,0001 y $0,08 \mu \mathrm{g} / \mathrm{m}^{2} / \mathrm{s}$. Los valores de concentración diarias de $\mathrm{NO}_{3}{ }^{-}$oscilaron entre 0,001 y $0,3 \mu \mathrm{g} / \mathrm{m}^{3}$ mientras que las deposiciones húmedas oscilaron entre $1 \times 10^{-5} \mu \mathrm{g} / \mathrm{m}^{2} / \mathrm{s}$ y $1 \times 10^{-2} \mu \mathrm{g} / \mathrm{m}^{2} / \mathrm{s}$.

En cuanto a los valores medios diarios del $\mathrm{SO}_{2}$ estos oscilan entre $1 \mu \mathrm{g} / \mathrm{m}^{3}$ y $50 \mu \mathrm{g} / \mathrm{m}^{3}$ con máximos de hasta $69 \mu \mathrm{g} /$ $\mathrm{m}^{3} \mathrm{y}$ valores de deposiciones secas medios diarios entre $1 \times 10^{-8}$ $\mu \mathrm{g} / \mathrm{m}^{2} / \mathrm{s}$ y $0,5 \mu \mathrm{g} / \mathrm{m}^{2} / \mathrm{s}$ y las deposiciones húmedas entre $1 \times 10^{-6}$ $\mu \mathrm{g} / \mathrm{m}^{2} / \mathrm{s}$ y $1 \mu \mathrm{g} / \mathrm{m}^{2} / \mathrm{s}$. Los valores de concentración diarias de $\mathrm{SO}_{4}{ }^{2-}$ oscilaron entre 0,0001 y $0,5 \mu \mathrm{g} / \mathrm{m}^{3}$ y sus deposiciones húmedas entre $1 \times 10^{-6} \mu \mathrm{g} / \mathrm{m}^{2} / \mathrm{s}$ y $0,05 \mu \mathrm{g} / \mathrm{m}^{2} / \mathrm{s}$.

Los valores de concentraciones diarias de $\mathrm{CO}$ en los 15 días simulados oscilaron entre $0,01 \mu \mathrm{g} / \mathrm{m}^{3}$ y $5,0 \mu \mathrm{g} / \mathrm{m}^{3}$ con 


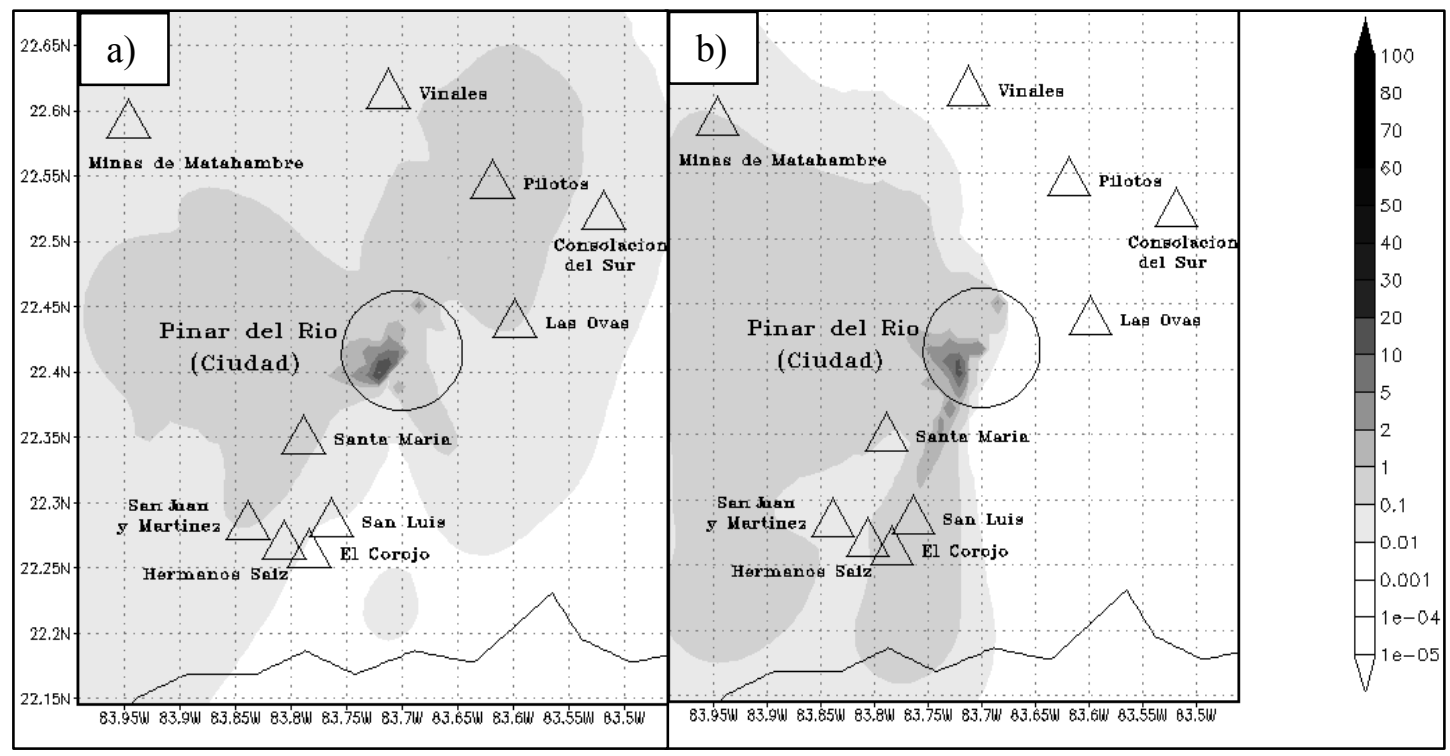

Figura 8 -Comportamiento medio diario de las concentraciones de $\mathrm{SO}_{2}$ los días 1 de agosto del 2007 (a) y 18 de julio del 2007 (b) .

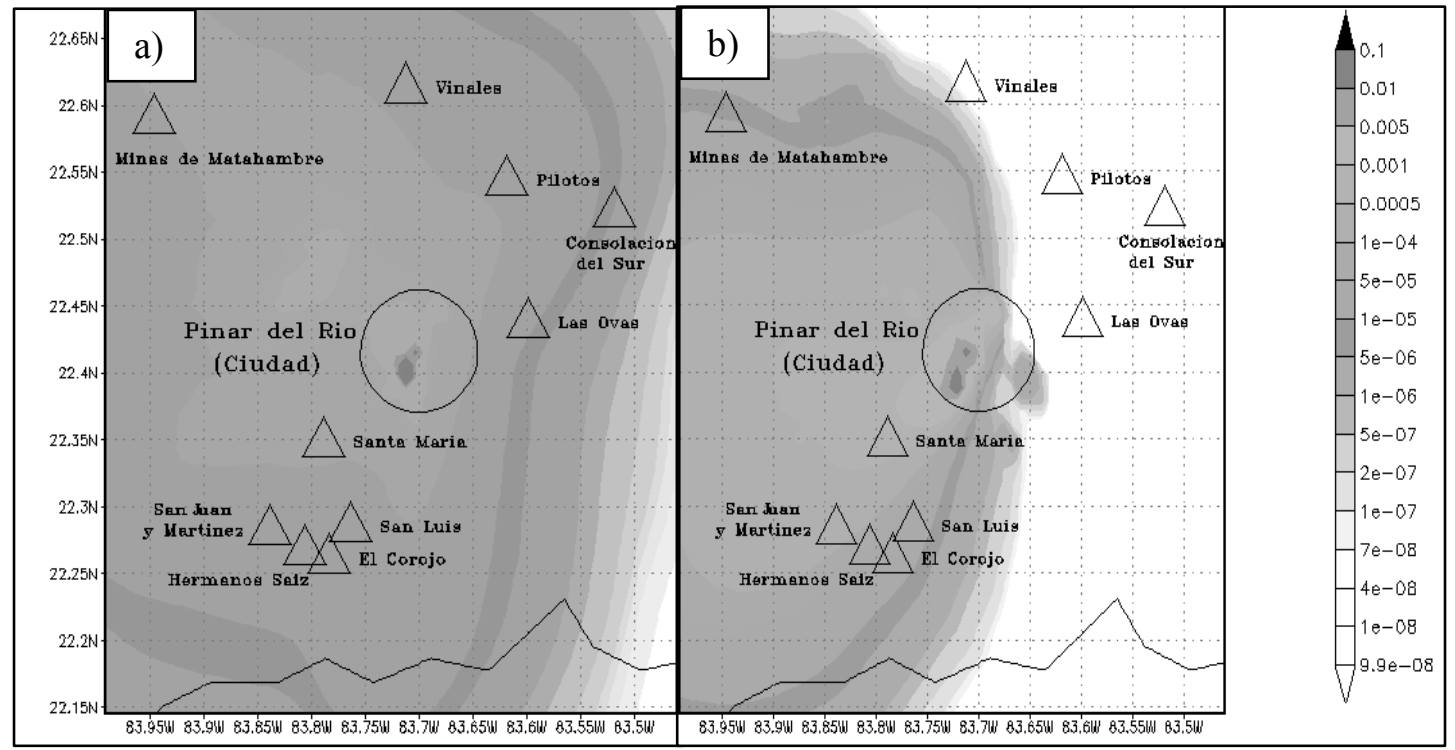

Figura 9 -Comportamiento medio diario de las deposiciones secas de $\mathrm{PM}_{10}$ los días 7 de julio del 2007 (a) y 12 de agosto del 2007 (b).

máximos de $1,7 \mu \mathrm{g} / \mathrm{m}^{3}$ mientras que los valores medios de concentraciones diarias de $\mathrm{PM}_{10}$ oscilaron entre $0,01 \mu \mathrm{g} / \mathrm{m}^{3}$ y $8,0 \mu \mathrm{g} / \mathrm{m}^{3}$ con máximos de $1,6 \mu \mathrm{g} / \mathrm{m}^{3}$ y deposiciones secas que oscilaron entre $1 \times 10^{-8} \mu \mathrm{g} / \mathrm{m} 2 / \mathrm{s} \mathrm{y} 0,1 \mu \mathrm{g} / \mathrm{m} 2 / \mathrm{s}$. Los valores de concentraciones diarias de $\mathrm{PM}_{25}$ oscilaron entre $1 \times 10^{-5} \mu \mathrm{g} /$ $\mathrm{m}^{3}$ y $3,0 \mu \mathrm{g} / \mathrm{m}^{3}$ con máximos de $0,8 \mu \mathrm{g} / \mathrm{m}^{3}$ mientras que sus deposiciones secas oscilan entre $1 \times 10^{-8} \mu \mathrm{g} / \mathrm{m}^{2} / \mathrm{s}$ y $0,0001 \mu \mathrm{g} /$ $\mathrm{m}^{2} / \mathrm{s}$.

Con el objetivo de determinar si las emisiones sobrepasan los valores guías para periodos de 24 horas para cada una de las especies simuladas son analizados los máximos valores de 24 horas registrados. Estos valores constituyen el máximo valor que se reportó en cada período de 24 horas por lo que si sobrepasan los valores guías significa que al menos una vez durante este día se incumplen las normas de calidad del aire. La Tabla 3 muestra el resumen de los máximos de concentraciones para períodos de 24 horas y en la Tabla 4 se pueden observar la cantidad de días que los máximos sobrepasan los valores normados y el por ciento que estos representan del total de $\mathrm{NO}_{\mathrm{x}}$ y $\mathrm{SO}_{2}$ respectivamente, ya que como se puede notar en la Tabla 3 son estas las únicas especies que sobrepasan sus valores guías. 


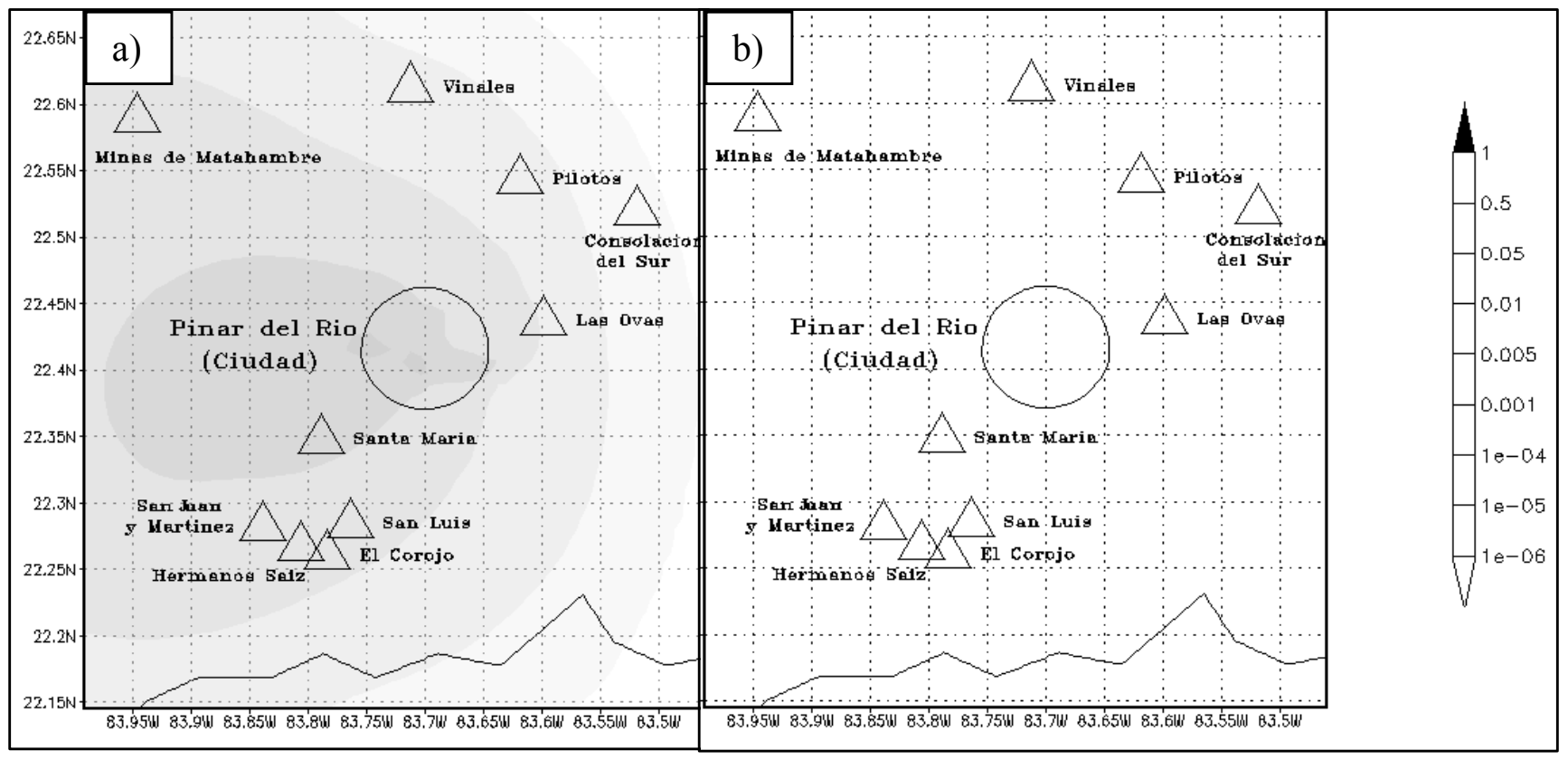

Figura 10 -Comportamiento medio diario de las deposiciones húmedas de $\mathrm{SO}_{4}{ }^{2-}$ los días 15 de julio del 2007 (a) y 27 de julio del 2007 (b).
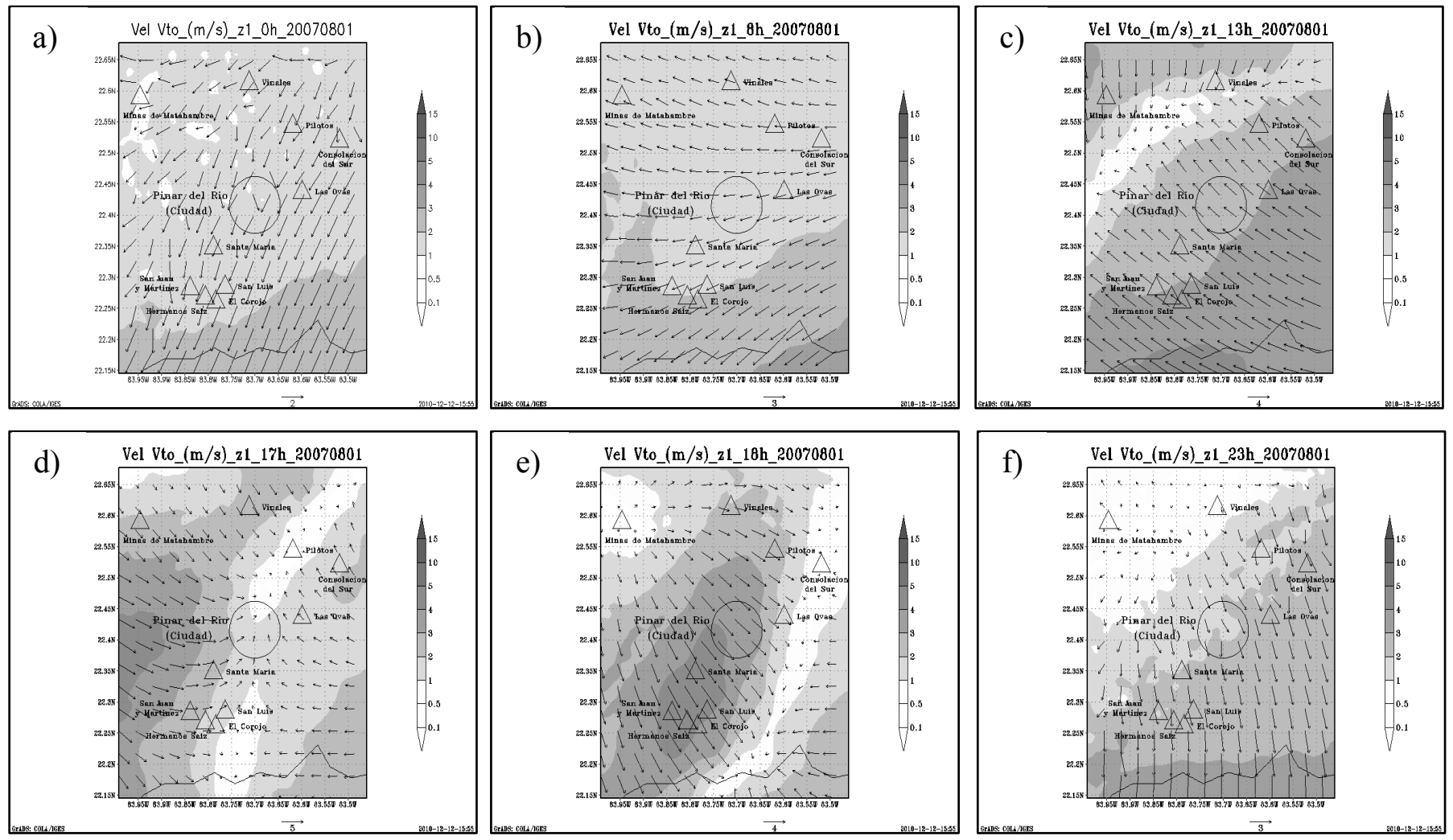

Figura 11 - Comportamiento medio horario de la dirección y fuerza del viento el día 1ro de agosto del 2007 a las 0 horas (a), 8 am (b), 1 pm (c), 5 pm (d), 6 pm (e) y 11 pm (f). 

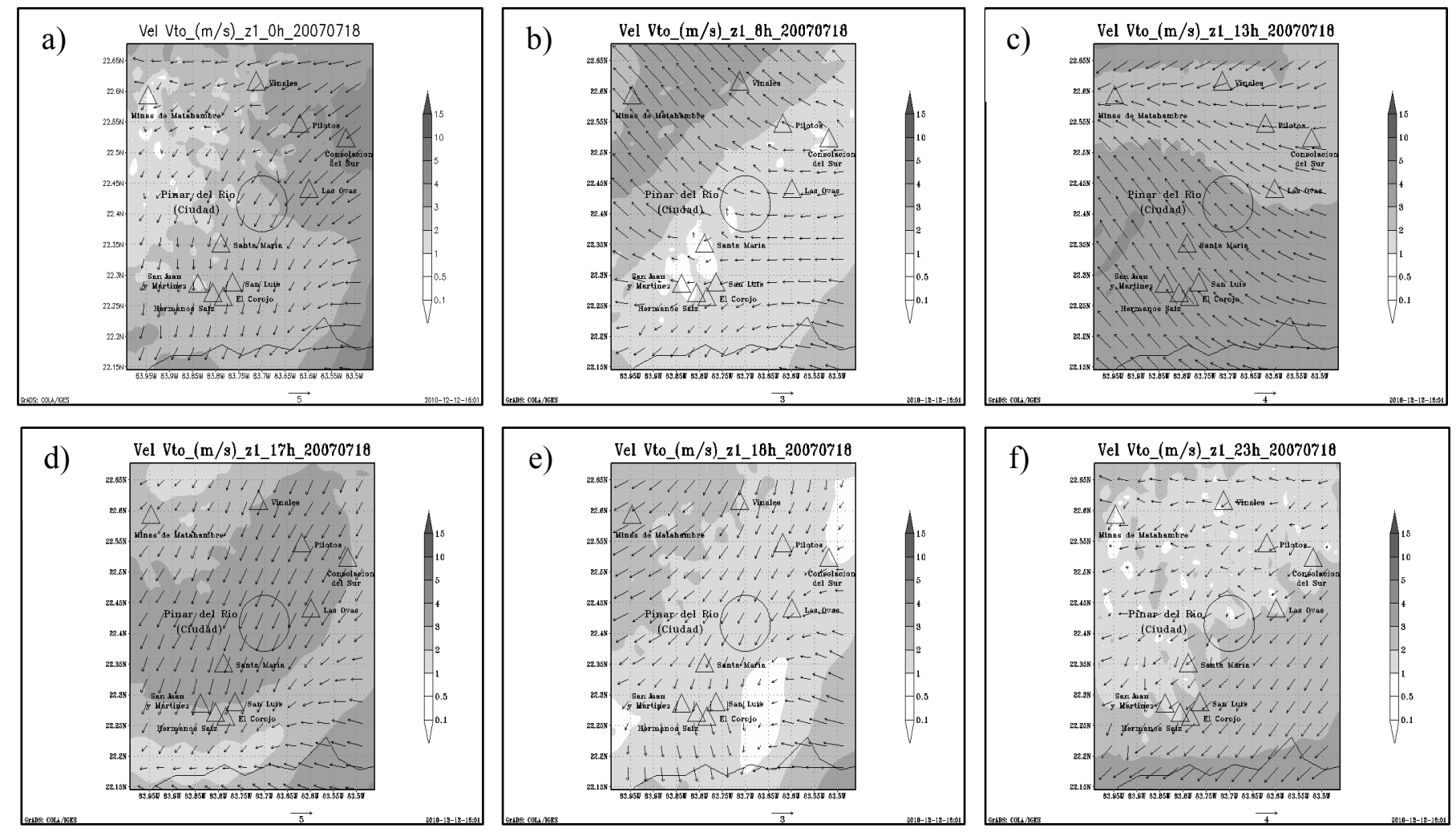

Figura 12 - Comportamiento medio horario de la dirección y fuerza del viento el día 18 de julio del 2007 a las 0 horas (a), 8 am (b), 1 pm (c), $5 \mathrm{pm}(\mathrm{d}), 6 \mathrm{pm}(\mathrm{e})$ y $11 \mathrm{pm}(\mathrm{f})$.

Tabla 3 - Valores máximos para período de 24 horas de $\mathrm{NO}_{\mathrm{x}}, \mathrm{SO}_{2}, \mathrm{CO}, \mathrm{PM}_{10}$ y $\mathrm{PM}_{25}$. $\left(\mathrm{En} \mu \mathrm{g} / \mathrm{m}^{3}\right)$.

\begin{tabular}{|c|c|c|c|c|c|c|c|c|c|c|c|}
\hline Días & $\mathbf{N O}_{\mathbf{x}}$ & $\mathbf{S O}_{\mathbf{2}}$ & $\mathbf{C O}$ & $\mathbf{P M}_{\mathbf{1 0}}$ & $\mathbf{P M}_{\mathbf{2 5}}$ & Días & $\mathbf{N O}_{\mathbf{x}}$ & $\mathbf{S O}_{\mathbf{2}}$ & $\mathbf{C O}$ & $\mathbf{P M}_{\mathbf{1 0}}$ & $\mathbf{P M}_{\mathbf{2 5}}$ \\
\hline $\mathbf{1 / 8 / 2 0 0 7}$ & 19,5 & 15,1 & 0,09 & 0,36 & 0,23 & $\mathbf{1 3 / 7 / 2 0 0 7}$ & 92,4 & 69,1 & 0,20 & 1,69 & 0,50 \\
\hline $\mathbf{2 / 8 / 2 0 0 7}$ & 23,1 & 17,8 & 0,10 & 0,47 & 0,13 & $\mathbf{1 4 / 7 / 2 0 0 7}$ & 32,9 & 24,9 & 0,14 & 0,66 & 0,22 \\
\hline $\mathbf{7 / 7 / 2 0 0 7}$ & 25,8 & 21,9 & 0,33 & 0,61 & 0,27 & $\mathbf{1 5 / 7 / 2 0 0 7}$ & 23,0 & 17,4 & 1,55 & 0,46 & 0,21 \\
\hline $\mathbf{8 / 7 / 2 0 0 7}$ & 43,7 & 34,1 & 1,78 & 1,10 & 0,85 & $\mathbf{1 7 / 7 / 2 0 0 7}$ & 16,4 & 12,1 & 0,37 & 0,46 & 0,30 \\
\hline $\mathbf{1 1 / 8 / 2 0 0 7}$ & 21,3 & 16,0 & 0,77 & 0,41 & 0,15 & $\mathbf{1 8 / 7 / 2 0 0 7}$ & 16,3 & 12,8 & 0,16 & 0,46 & 0,26 \\
\hline $\mathbf{1 2 / 8 / 2 0 0 7}$ & 20,8 & 15,9 & 0,34 & 0,50 & 0,39 & $\mathbf{2 7 / 7 / 2 0 0 7}$ & 17,5 & 13,8 & 0,15 & 0,34 & 0,22 \\
\hline $\mathbf{1 2 / 7 / 2 0 0 7}$ & 33,8 & 25,7 & 0,29 & 0,60 & 0,21 & $\mathbf{2 8 / 7 / 2 0 0 7}$ & 17,8 & 14,8 & 1,04 & 0,39 & 0,35 \\
\hline & & & & & & $\mathbf{3 0 / 8} / \mathbf{2 0 0 8}$ & 17,7 & 13,3 & 0,18 & 0,39 & 0,19 \\
\hline
\end{tabular}

\subsection{Comportamiento de los máximos valores horarios registrados}

Con el objetivo de determinar si las emisiones sobrepasan los valores guías para periodos de 1 hora para cada una de las especies simuladas son analizados los máximos valores horarios registrados. Estos valores constituyen el máximo valor horario que se reportó diariamente por lo que si sobrepasan estos valores significa que al menos una vez durante este día se sobrepasaron los valores regulatorios. De todas las especies simuladas solo sobrepasaron los valores guías establecidos los $\mathrm{NO}_{\mathrm{x}}$ y $\mathrm{SO}_{2}$.

\subsubsection{Máximos valores horarios registrados de $\mathrm{NO}_{\mathrm{x}}$.}

A los efectos regulatorios se adopta el criterio de considerar todos los $\mathrm{NO}_{\mathrm{x}}$ emitidos en chimenea como $\mathrm{NO}_{2}$ ya que inmediatamente después del enfriamiento del efluente gaseoso, la mayor parte de los óxidos de nitrógeno formados, 
Tabla 4 - Cantidad de días en los que los máximos para períodos de 1 hora y 24 horas sobrepasan los valores guías de $\mathrm{NO}_{\mathrm{x}}$ y $\mathrm{SO}_{2}$.

\begin{tabular}{|c|c|c|c|c|c|c|}
\hline \multirow{5}{*}{$\begin{array}{l}\text { Períodos } \\
\text { de } 1 \\
\text { hora }\end{array}$} & \multicolumn{3}{|l|}{$\mathrm{NO}_{\mathrm{x}}$} & \multicolumn{3}{|l|}{$\mathrm{SO}_{2}$} \\
\hline & $\begin{array}{l}\text { Máximos por encima del } \\
\text { valor... }\end{array}$ & \# Días & $\%$ & $\begin{array}{l}\text { Máximos por encima del } \\
\text { valor... }\end{array}$ & \# Días & $\%$ \\
\hline & $\begin{array}{l}\text {...propuesto como CMA para } \\
\text { Cuba. }\end{array}$ & 14 & $93 \%$ & $\begin{array}{l}\text {...propuesto como CMA } \\
\text { para Cuba. }\end{array}$ & 3 & $20 \%$ \\
\hline & ...estándar EPA. & 11 & $73 \%$ & ...estándar EPA. & 6 & $40 \%$ \\
\hline & ...recomendado por la OMS. & 9 & $60 \%$ & & & \\
\hline \multirow{3}{*}{$\begin{array}{l}\text { Períodos } \\
\text { de } 24 \\
\text { horas }\end{array}$} & $\begin{array}{l}\text { Máximos por encima del } \\
\text { valor... }\end{array}$ & \# Días & $\%$ & $\begin{array}{l}\text { Máximos por encima del } \\
\text { valor... }\end{array}$ & \# Días & $\%$ \\
\hline & ...de CMA para Cuba. & 2 & $13 \%$ & ...de CMA para Cuba. & 1 & $6 \%$ \\
\hline & & & & $\begin{array}{l}\text {...recomendado por la } \\
\text { OMS. }\end{array}$ & 5 & $33 \%$ \\
\hline
\end{tabular}

Tabla 5 - Valores máximos horarios de $\mathrm{NO}_{\mathrm{x}}\left(\mathrm{en} \mu \mathrm{g} / \mathrm{m}^{3}\right)$.

\begin{tabular}{|l|l|l|l|l|l|l|l|l|}
\hline Días & Máx. & Hora & Días & Máx. & Hora & Días & Máx. & Hora \\
\hline $\mathbf{1 / 8 / 2 0 0 7}$ & 326 & $7 \mathrm{pm}$ & $\mathbf{1 2 / 8 / 2 0 0 7}$ & 163 & $3 \mathrm{pm}$ & $\mathbf{1 7 / 7 / 2 0 0 7}$ & 240 & $1 \mathrm{pm}$ \\
\hline $\mathbf{2 / 8 / 2 0 0 7}$ & 199 & $8 \mathrm{am}$ & $\mathbf{1 2 / 7 / 2 0 0 7}$ & 187 & $6 \mathrm{pm}$ & $\mathbf{1 8 / 7 / 2 0 0 7}$ & 249 & $7 \mathrm{pm}$ \\
\hline $\mathbf{7 / 7 / 2 0 0 7}$ & 294 & $6 \mathrm{pm}$ & $\mathbf{1 3 / 7 / 2 0 0 7}$ & 1077 & $5 \mathrm{pm}$ & $\mathbf{2 7 / 7 / 2 0 0 7}$ & 273 & $9 \mathrm{am}$ \\
\hline $\mathbf{8 / 7 / 2 0 0 7}$ & 817 & $7 \mathrm{am}$ & $\mathbf{1 4 / 7 / 2 0 0 7}$ & 233 & $6 \mathrm{pm}$ & $\mathbf{2 8 / 7 / 2 0 0 7}$ & 190 & $7 \mathrm{pm}$ \\
\hline $\mathbf{1 1 / 8 / 2 0 0 7}$ & 457 & $5 \mathrm{pm}$ & $\mathbf{1 5 / 7 / 2 0 0 7}$ & 127 & $6 \mathrm{pm}$ & $\mathbf{3 0 / 8 / 2 0 0 8}$ & 174 & $11 \mathrm{pm}$ \\
\hline
\end{tabular}

están presentes como monóxido (NO), pero esta especie se oxida rápidamente a $\mathrm{NO}_{2}$ por acción del $\mathrm{O}_{3}$, por lo que se comparan los resultados de $\mathrm{NO}_{\mathrm{x}}$ con los valores normados para $\mathrm{NO}_{2}$.

En la Tabla 5 se observan los valores máximos horarios que se registraron durante los 15 días simulados mientras que la Tabla 4 constituye un resumen de la cantidad de días en los que estos valores sobrepasan los valores guías y el por ciento que representan del total. Aunque para períodos de 1 hora en nuestro país no se tiene aún una CMA resulta alarmante como el $93 \%$ de los días simulados presentan valores máximos por encima del valor propuesto. Como se observa en la Tabla 3 los horarios de la tarde son más propensos a la ocurrencia de estos máximos.

\subsubsection{Máximos valores horarios registrados de $\mathrm{SO}_{2}$}

Los valores máximos horarios que se registraron durante los 15 días simulados se exponen en la Tabla 6, mientras que la Tabla 4 es un resumen de la cantidad de días en los que estos valores sobrepasan los valores guías y el por ciento que representan del total. Esta especie presenta menos días con máximos que sobrepasan los valores guías, encontrándose por encima del valor propuesto para Cuba solo un $20 \%$. Son también los horarios de la tarde para esta especie los más proclives a la ocurrencia de máximos.

En las Figuras 13 y 14 es posible observar la posición de los máximos de $\mathrm{NO}_{\mathrm{x}}$ y $\mathrm{SO}_{2}$ respectivamente. Estos se dieron en cuatro puntos fundamentales los cuales se encuentran cercanos a las fuentes de emisión.

Como se puede observar en las Figuras 13 y 14 la zona sur-oeste de la ciudad es la más sensible a episodios de mala calidad del aire por ser esta donde se producen con mayor frecuencia los máximos de concentraciones superiores a los valores guías.

\section{CONCLUSIONES}

- Se pueden diferenciar dos patrones bien definidos del comportamiento medio horario de las concentraciones de todas las especies simuladas. Un patrón para el $\mathrm{SO}_{2}$ y $\mathrm{NO}_{\mathrm{X}}$ con 
Tabla 6 - Valores máximos horarios de $\mathrm{SO}_{2}\left(\mathrm{en} \mu \mathrm{g} / \mathrm{m}^{3}\right)$.

\begin{tabular}{|l|l|l|l|l|l|l|l|l|}
\hline Días & Máx. & Hora & Días & Máx. & Hora & Días & Máx. & Hora \\
\hline $\mathbf{1 / 8 / 2 0 0 7}$ & 243 & $7 \mathrm{pm}$ & $\mathbf{1 2 / 8 / 2 0 0 7}$ & 121 & $3 \mathrm{pm}$ & $\mathbf{1 7 / 7 / 2 0 0 7}$ & 181 & $1 \mathrm{pm}$ \\
\hline $\mathbf{2 / 8 / 2 0 0 7}$ & 150 & $8 \mathrm{am}$ & $\mathbf{1 2 / 7 / 2 0 0 7}$ & 141 & $6 \mathrm{pm}$ & $\mathbf{1 8 / 7 / 2 0 0 7}$ & 188 & $7 \mathrm{pm}$ \\
\hline $\mathbf{7 / 7 / 2 0 0 7}$ & 221 & $6 \mathrm{pm}$ & $\mathbf{1 3 / 7 / 2 0 0 7}$ & 811 & $5 \mathrm{pm}$ & $\mathbf{2 7 / 7 / 2 0 0 7}$ & 206 & $9 \mathrm{am}$ \\
\hline $\mathbf{8 / 7 / 2 0 0 7}$ & 613 & $7 \mathrm{am}$ & $\mathbf{1 4 / 7 / 2 0 0 7}$ & 175 & $6 \mathrm{pm}$ & $\mathbf{2 8 / 7 / 2 0 0 7}$ & 173 & $1 \mathrm{pm}$ \\
\hline $\mathbf{1 1 / 8 / 2 0 0 7}$ & 345 & $5 \mathrm{pm}$ & $\mathbf{1 5 / 7 / 2 0 0 7}$ & 171 & $5 \mathrm{pm}$ & $\mathbf{3 0 / 8 / 2 0 0 8}$ & 131 & $11 \mathrm{pm}$ \\
\hline
\end{tabular}

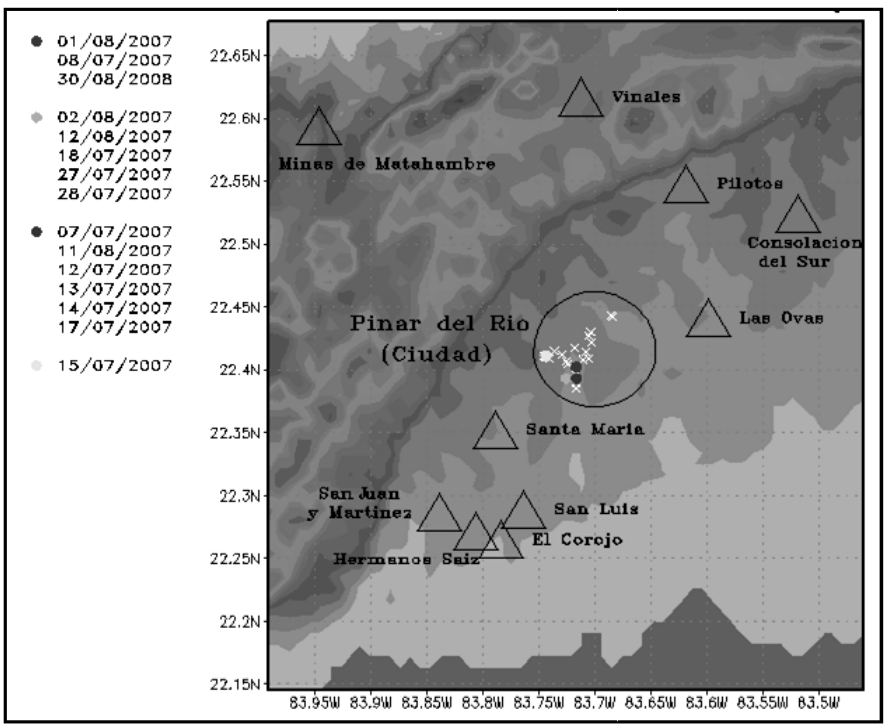

Figura 13 - Posición de los máximos de $\mathrm{NO}_{\mathrm{x}}$ durante los 15 días simulados.

máximos alrededor de las 8 am y 8 pm, y otro patrón para el $\mathrm{CO}, \mathrm{PM}_{10}, \mathrm{PM}_{25}, \mathrm{HNO}_{3}, \mathrm{NO}_{3}{ }^{-}$y $\mathrm{SO}_{4}{ }^{2-}$ con un máximo alrededor de las 7 am y un mínimo alrededor de las $4 \mathrm{pm}$.

- Los mayores valores medios horarios de deposición seca ocurren entre las 7 am y 8 am. Los valores medios horarios de deposición seca del $\mathrm{PM}_{10}$ tienden disminuir a partir de las 9 $\mathrm{pm}$ mientras que sus concentraciones tienen a aumentar.

- Los mayores valores medios horarios de deposición húmeda ocurren entre la 1 pm y 6 pm, en correspondencia con los horarios de mayor monto de precipitación.

- Las concentraciones medias diarias de todas las especies así como sus valores medios diarios de deposiciones secas y húmedas tienden a comportarse de dos formas. La primera es afectando a toda la rejilla y la segunda afectando solo la parte oeste de esta.

- Las únicas especies que sobrepasan los valores normados para períodos de 24 horas son el $\mathrm{SO}_{2}$ y $\mathrm{NO}_{\mathrm{X}}$, con máximos que alcanzan los $69,1 \mu \mathrm{g} / \mathrm{m}^{3}$ y $92,4 \mu \mathrm{g} / \mathrm{m}^{3}$ respectivamente.

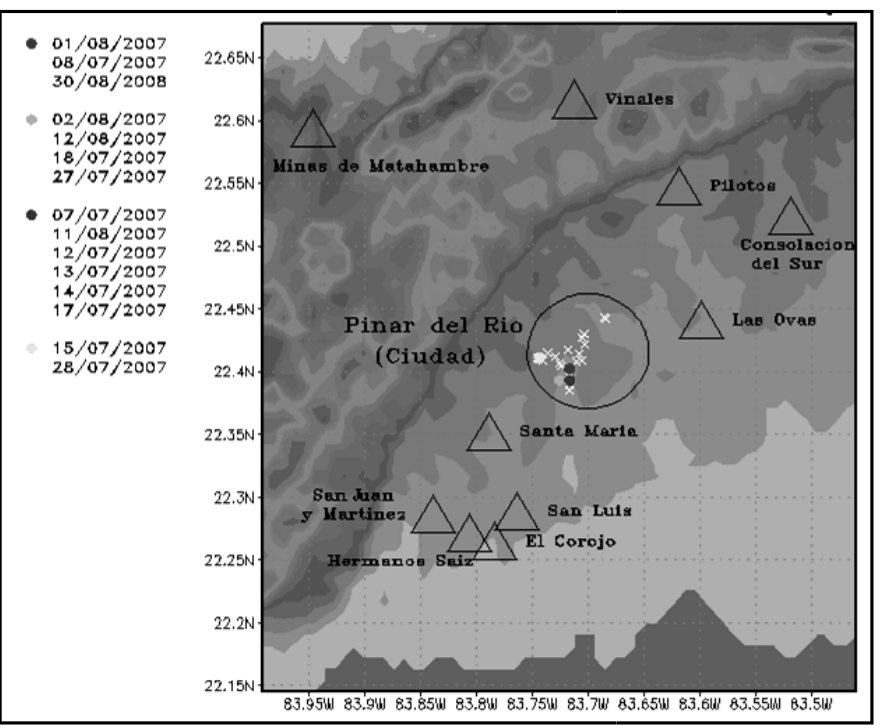

Figura 14 - Posición de los máximos de $\mathrm{SO}_{2}$ durante los 15 días simulados.

- Las únicas especies que sobrepasan los valores normados para períodos de 1 hora son el $\mathrm{SO}_{2}$ y $\mathrm{NO}_{\mathrm{X}}$, con máximos que alcanzan los $811 \mu \mathrm{g} / \mathrm{m}^{3}$ y $1077 \mu \mathrm{g} / \mathrm{m}^{3}$ respectivamente, localizándose estos máximos en cuatro puntos fundamentales cercanos de las fuentes de emisión, derivando en que sea la zona sur-oeste de la ciudad la más sensible a episodios de mala calidad del aire.

\section{RECOMENDACIONES}

- Realizar la simulación utilizando los datos de todas las fuentes puntuales no solo de la ciudad de Pinar del Río, sino hacer extensivo el estudio para toda la provincia.

- Hacer extensiva la simulación para días del período poco lluvioso.

- Continuar profundizando y realizar estudios de sensibilidad para establecer las causas de las diferencias encontradas entre los máximos de concentración y de deposición seca para el $\mathrm{PM}_{10}$. 


\section{REFERENCIAS BIBLIOGRÁFICAS}

BUILTJES, P.: Major twentieth century milestones in air pollution modelling and its application. Earth and Environmental Science. Air pollution modeling and its application XIV. Part 1, p 3-16, 2001.

COLLAZO, A.: Análisis de la contaminación transfronteriza y local de los compuestos gaseosos precursores de la deposición ácida húmeda y formación de ozono en Cuba. p 58-110, 2011.

COLLAZO, A et al.: Modelación de las transformaciones químicas a escala local del $\mathrm{SO}_{2}$ a sulfato. Resultado Programa Ramal Científico Técnico Protección del medio ambiente y el desarrollo Sostenible cubano. Subprograma. Previsión, prevención y mitigación de impactos Ambientales de la contaminación atmosférica y sus Variables físicas conexas. Proyecto 0030230, p 15-80, 2004.

FONSECA, M.: Caracterización de las concentraciones de los compuestos gaseosos del nitrógeno atmosférico asociados a diversos Tipos de Situaciones Sinópticas (TSS) en la estación La Palma, Pinar del Río, Cuba, p 135, 2010.

FONSECA, Y.: Implementación y aplicación del sistema de modelación CALMET-CALPUFF-CALPOST a escala local. p 32-46, 2010.

GONZÁLEZ, Y.: Estudio preliminar de la eficiencia y efectividad de los generadores terrestres de yoduro de plata en Cuba. p 20-68, 2010

HAO, J et al.: Air quality impacts of power plant emissions in Beijing. Environmental Pollution.Volume, v.147, n. 2. p 401-408, 2006.

LEVY, $\mathrm{J}$ et al.: Using CALPUFF to evaluate the impacts of power plant emissions in Illinois: model sensitivity and implications. Atmospheric Environment, v. 36, n. 6, p 1063-1075, 2001.
ONE: Datos de la Oficina Nacional de Estadísticas de Cuba. 2009. Disponible en: http://www.one.cu/aed2009/01Pinar del Río/Municipios/11Pinar del Río/Index.htm

RAMÍREZ, J.: Estudio de las variaciones en las concentraciones de ozono troposférico en Cuba y su vinculación con algunos fenómenos meteorológicos. Tesis para la obtención del grado científico de candidato a Doctor en Ciencias Geográficas, La Habana, p 15-54, 1989.

RODRÍGUEZ, D. et al.: Sistema Automatizado de Gestión de Información de Fuentes Contaminantes (SAGIFC). Publicación electrónica del IV Congreso Cubano de Meteorología. ISBN: 978-959-7167-12-9. La Habana, Centro de Convenciones Capitolio, Cuba. p 7-18, 2007.

SÁNCHEZ, P et al.: Impactos de la contaminación atmosférica en la salud en el municipio Habana Vieja. Resultado Programa Ramal Científico Técnico Protección del medio ambiente y el desarrollo Sostenible cubano. Subprograma. Previsión, prevención y mitigación de impactos Ambientales de la contaminación atmosférica y sus Variables físicas conexas. Proyecto 3114, p 13-63, 2004.

SEHMEL, G.A.: Particle and gas dry deposition - a review. Atmospheric Environment. Volume 14, p 983-1011, 1980. VALDÉS-MANZANILLA, A et al. (): Simulación de la concentración de $\mathrm{SO}_{2}$ emitido por fuentes fijas mayores durante 2003 en el noroeste de Chiapas y centro de Tabasco, México. Revista Internacional de Contaminación Ambiental.. ISSN: 0188-4999, v. 24, n. 2, pp 71-77, 2008. WHO: The World Health Report 2000. Health Systems: Improving Performance. ISBN: 924156198 X. ISNN: 1020-3311, 2000. 